\title{
Design of Decision-making System for Fire-fighting and Rescue Aid Based on GIS
}

\author{
Qiuhong Pei \\ Chinese People's Armed Police Force Academy \\ peiqh@sina.com
}

Keywords: GIS geographic information system; fire-fighting plan; aid decision making; Path selection

\begin{abstract}
With the unceasing thorough development of reform and open policy and rapid development of economic construction, high-rise buildings, petrochemical industry and public places grow with each passing day. The urban development increasingly diversified and complicated, and the factors for causing a fire are also increasing rapidly. Facing the complicated regional environment, the system will be used to solve the problems the fire troops will encounter in the fire and emergency rescue. This system focuses on utilizing GIS geographic information system for its unique spatial analysis function and visual expression of the map. The use of visual studio can help to gain the best path for fire troops to go out of fire police, the division of damage scope, the search for the water nearby and the call of fire-fighting plan. Meanwhile, fire-fighting plan can be exhibited in the three-dimensional vision through Google sketch up. We could provide the sufficient reference information for the combatants at the grass roots to assist with the temporary commanding, and help to make scientific decisions.
\end{abstract}

\section{Introduction}

Fire fighting and rescue aided decision system based on GIS using the spatial visualization, spatial orientation function react on the GIS map clearly such as area map, roads within the jurisdiction of distribution, the main building information distribution, the key unit of fire, water, fire hydrant and fire squadron It is convenient for the auxiliary fire command personnel to obtain the unidirectional information of the fire-fighting command, so as to make it easier for the central teams in the district to be familiar with the road of the district and the distribution of key units.

The properties of buildings in GIS maps remind the police personnel the stored material, historical fire data, their level of risk size, scope of hazardous chemicals leakage accident of influence and fire-fighting preplan calls and stereoscopic display. It is convenient for the commander to arrange tasks or call reinforcements on the way out [1].

\section{System demand analysis}

\subsection{The main problems}

(1)Most of the police in the grass-roots units use the fire fighting experience or intuition of the commander to rescue the fire.

(2)There is a certain blindness and lack of scientific basis.

(3)Urban electronic maps such as baidu map and gaud map, etc., do not include fire information.

\subsection{The basic unit's demand for fire - extinguishing aid system}

(1)The existing units of urban areas, key units of fire fighting, the fighting power of fire fighting in districts, urban fire fighting facilities, public places and water distribution information are reflected in the GIS platform(Set the property value: whether the building is fire key unit, personnel situation, building nature, the fire alarm statistics of the building, the personnel equipment of the fire fighting force, etc) 
(2)The best way to locate and select fire squadron is to locate the fire location through the information provided by the alarm.

(3)In the case of fire in the building, the relevant squadron should be prepared in advance by adjusting the attribute value of the building.

(4)If there is a bigger fire, there are more forces. The fire field commander can use the system to divide the fire fighting area of each unit and find out the recent distribution of fire supplies through the property query, so as to facilitate the call of fire-fighting materials.

(5)The corresponding plan management can be carried out in GIS map[2]..

\section{Overall designs}

\subsection{System operating environment requirements}

Operating system: Windows 7 and above.

Development tools: Visual Studio 2008, Google sketch up 8, ARCGIS 9.3, ARCGIS engine 9.3 etc.

\subsection{System structure design}

The system is mainly based on GIS map, through the visual studio using dijkstra algorithm to calculate the shortest path, and add the roadblock according to the actual situation to realize the path selection function. The cache area analysis function of ARCGIS is used to devise warning areas for hazardous accidents. The hazard level is assessed by setting the building properties of the GIS map to display the material stored in the disaster site. The function of the fire extinguishing plan is realized by connecting to a Word document by hyperlink or by using a $3 \mathrm{~d}$ fire-fighting plan made by Google sketch up[3].

\section{3. module division}

According to the design of the system and the functions of the system, four main modules are classified. Figure 1 is a map of modules.

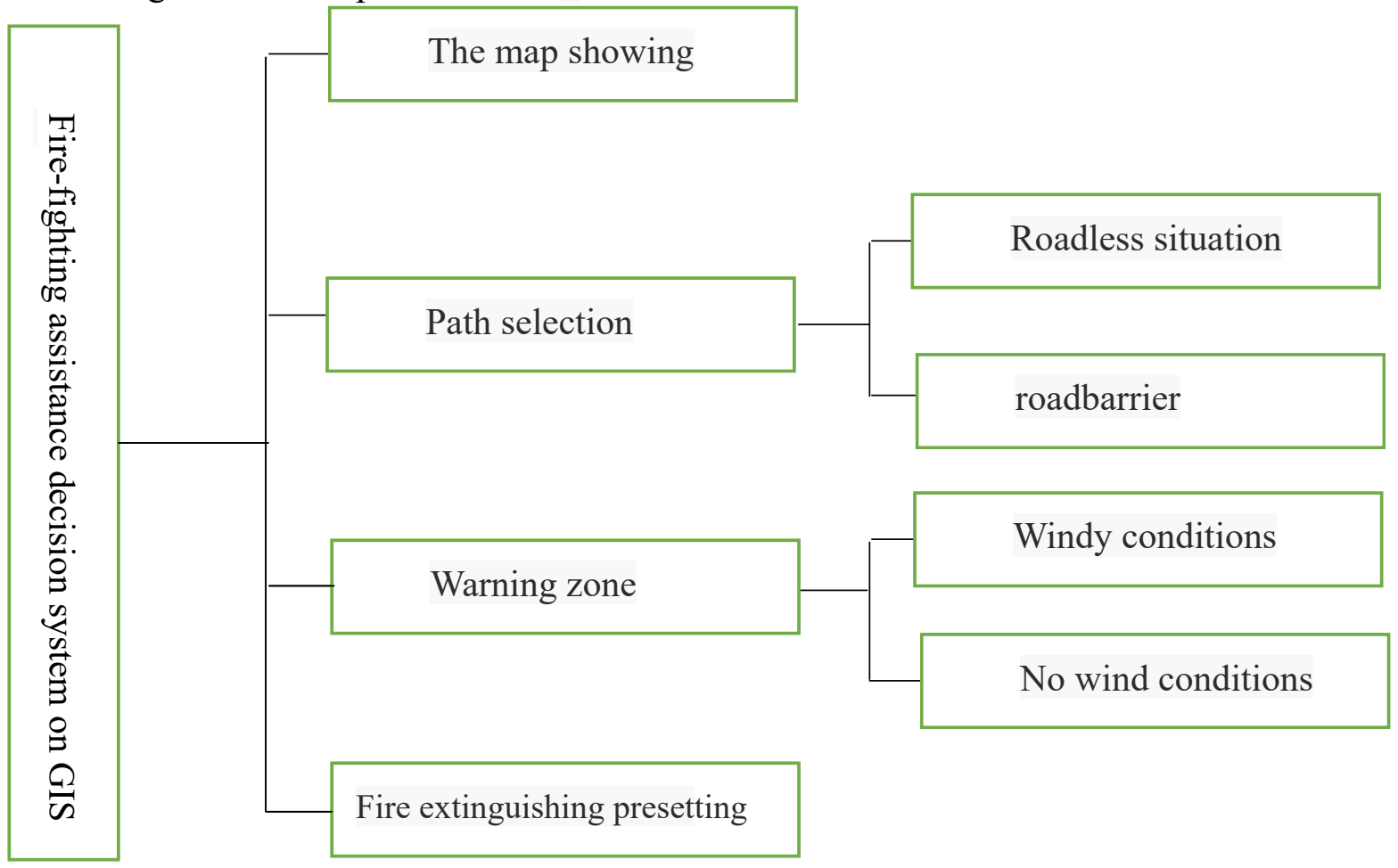

Figure 1 System block diagram 


\subsection{Design of map elements}

Considering the shortest path problem two geographic databases are created. The path layer and the road node layer are set in the file geographic database to facilitate the path calculation. Other layers are set in a personal geographic database.

A file geographic database is a collection of GIS data sets through folders, which can store, query, and manage spatial data. It can be used by multiple users simultaneously, but the same data can only be edited by one user.

The personal geographic database stores all data sets in Microsoft access data files. The spatial data and non-spatial data are stored and managed. Only one user can edit and modify it at a time.

This system layer attribute table includes: Path layer table, Road node layer table, Hydrant layer table, Supermarket layer table, Park layer table, Community layer table, School layer table, Residential layer table, Waters layer table, Green belt layer table, The regional layer table etc.

\section{Detailed design and implementation}

\subsection{Interface design}

Through Visual Studio 2008, the project is set up to select the GIS functions that need to be called and design the system interface.

\subsection{Grassroots geographic data establishment}

Based on ARCGIS software, the city map is used to perform vectoring operation through urban image data. First, we set up a geographic database through ArcCatalog, and set appropriate factor attributes, and add fire data to map building elements. The ArcMap is used to make the image data and input the attribute value. Provide basic data platform for system operation.

\subsection{Visual Studio program call}

(1) Program function namespace call

(2) Loading the map

(3) The shortest path calculation code is as follows: private void buttonItem8_Click(object sender, EventArgs e) $\{\ldots$

string startnode $=$ twonodecol.NodeCol[0].NodeID;

string endnode $=$ twonodecol.NodeCol[1].NodeID;

Plan( nodeCollection, startnode);

ArrayList arylist $=$ getMinDistancePath(endnode);

if (arylist.Count $>1)$

$\{$ textBox1.Text $=$ " Shortest path result:"; \}

else

\{ MessageBox.Show("There is no path!"); \}

double juli $=0.0000$;

double distance $=0.00$;

for (int $\mathrm{j}=0 ; \mathrm{j}<$ arylist.Count $; \mathrm{j}++$ )

$\{$ if $(\mathrm{j}<$ arylist.Count -1$)$

$\{$ IPoint pt1

_nodeCollection[Convert.ToString(arylist[j])].Point; IPoint pt2

_nodeCollection[Convert.ToString(arylist $[\mathrm{j}+1])]$.Point;

IPolyline pline $=$ new PolylineClass () ;

pline.FromPoint $=p t$;

pline. ToPoint $=p t 2$;

string nodelid

_nodeCollection[Convert.ToString(arylist[j])].NodeID; 


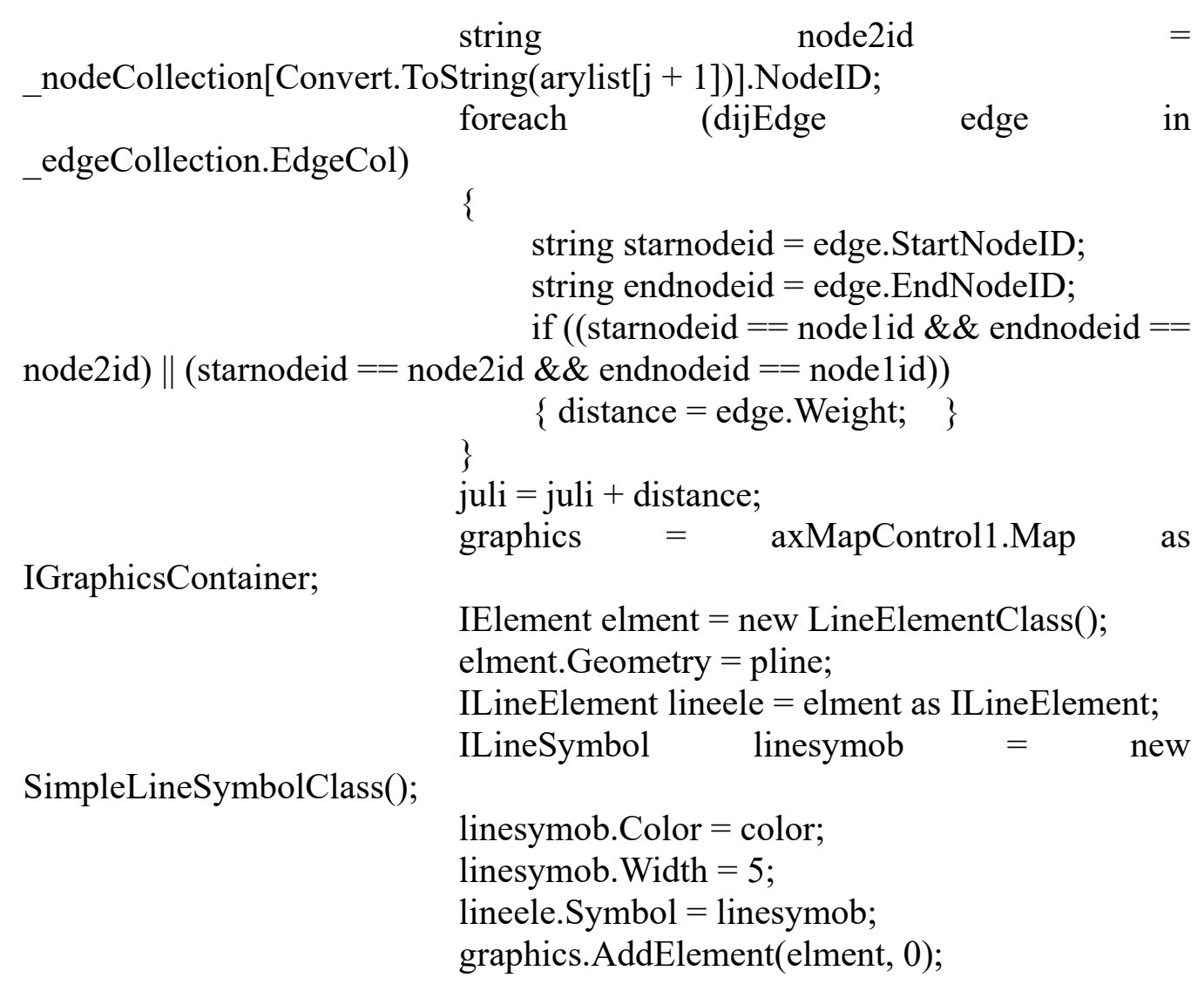

axMapControl1.Refresh(esriViewDrawPhase.esriViewGraphics, null, null);

ISimpleLineSymbol simplelinebol = new SimpleLineSymbolClass(); axMapControl1.FlashShape(pline, 3, 200, simplelinebol as ISymbol); string result $=$ Convert.ToString(arylist[j]) + "----->" + Convert.ToString(arylist $[\mathrm{j}+1])+$ ", ";

axMapControl1.Refresh(esriViewDrawPhase.esriViewGraphics, null, null);

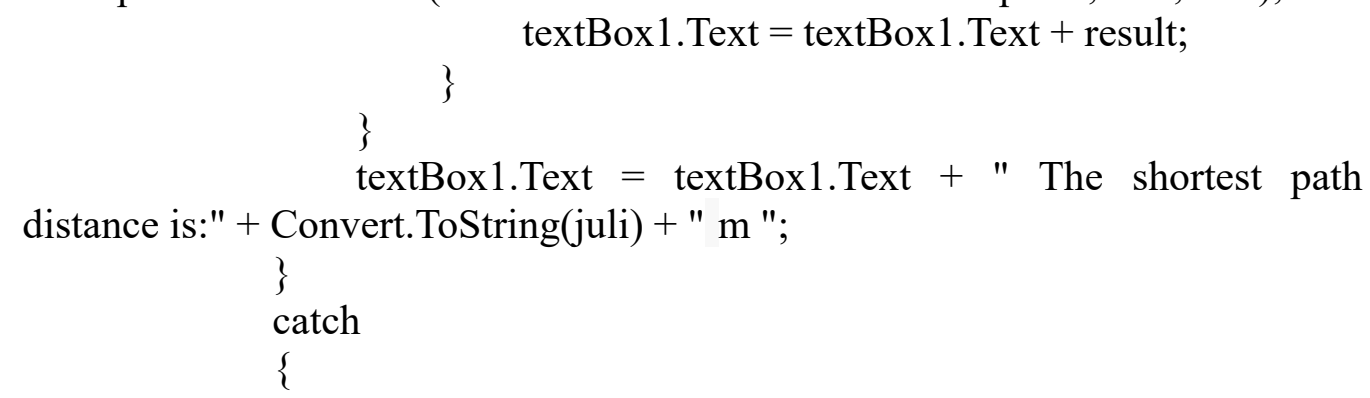
textBox1.Text $=$ textBox1.Text + result;

MessageBox.Show("Analysis error! Make sure you build the network correctly!");

(4) Setting up roadblocks

Roads could be built or heavily congested. To increase the robustness of the system, the system has designed the roadblock function. The node is no longer considered when the system is calculated by setting up a roadblock at the node.

(5) Color Settings

In the case of complex forces scheduling, multiple squadrons are required. Or, if you need to dispatch reinforcements, there are often paths that require multiple routes. The color setting option is designed, and the line color can be adjusted by setting R, G, and B values. 
(6) Updating the road

Urban road information will have road maintenance and so on. The road information needs to be modified. The calculation result of the alarm path is adjusted by changing the weight of the road.

(7) Location of accident location

When an officer receives a call, he or she needs to make a choice of route and must locate the location in the map. The location of the location of the accident can be located by using the attribute query function of GIS.

(8) Property inquiry and fire response plan call

Police personnel need to understand the accident site construction information, using GIS risk comes to the features of the attribute query; view the building fire-fighting preplan, familiar with the role assignment of fire fighting.

(9) Analysis of pollution

There will be wind and no wind in the leakage of dangerous chemicals. In the case of wind free, it is easier to determine the radius of influence based on the amount of dangerous chemicals. In the case of wind, the wind level and wind direction need to be determined [4]. The cache area is determined by GIS.

\subsection{Google sketch up8 Fire planning}

The Google sketch up8 is a convenient and intuitive three-dimensional mapping software. Through the Google sketch up8, a three-dimensional display of the fire-fighting plan can be carried out, so that the personnel in the fire-fighting and rescue can be familiar with their position at all times[5].

(1) Model making

The Google sketch up8 can be called module, which can be made by module and can be made in $3 \mathrm{~d}$ plan.

(2) Three dimensional display of fire-fighting plan

After the modules are finished, they can be combined and assembled to carry out the corresponding fire-fighting plan. See figure 2.

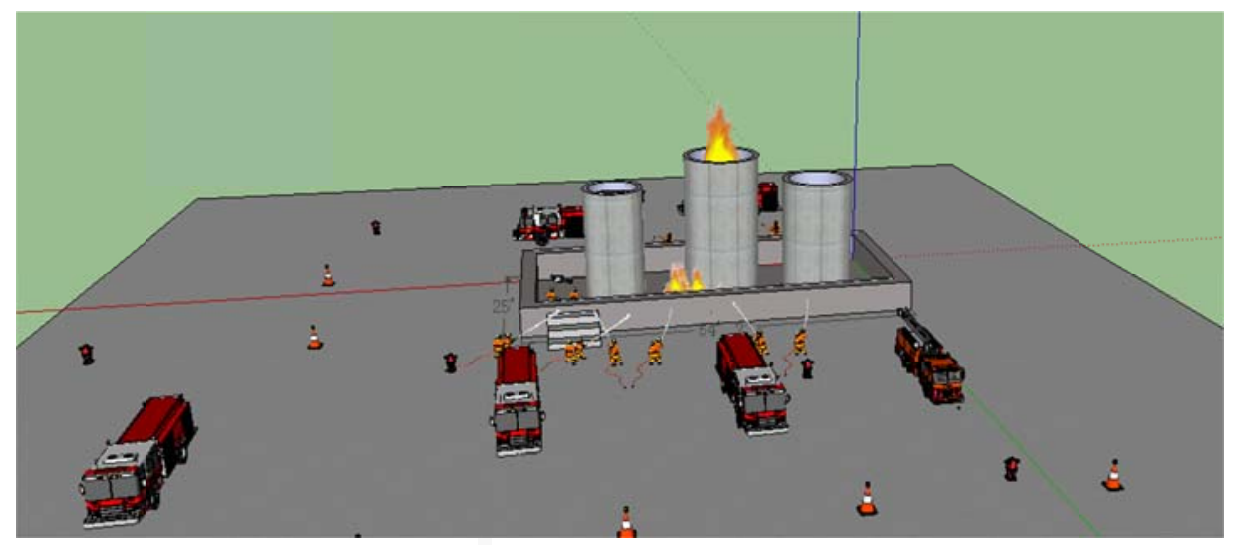

Figure 2 Three dimensional display of fire-fighting plan

\subsection{Fire extinguishing plan text display}

In addition to the three-dimensional intuitive fire suppression plan display, specific text description is required to clarify the task assignment of each rescue unit. Also through GIS hyperlink to carry out the fire plan text calls.

\section{Conclusions}

The system is based on GIS geographic information system platform development, which is an auxiliary decision-making system for firefighting units in fire fighting. The advantages are as follows:

(1) Easy and easy to use. 
(2) Information on the contents of the map, especially on fire protection.

(3) The location of the accident location and the selection of the best alarm route.

(4) The basic information of the building of the site can be mastered.

(5) Fire fighting plan calling.

System deficiencies are as follows:

(1) System response is slower

(2) There are some unreasonable designs of data attributes

(3) There is a certain redundancy in the program.

\section{References}

[1]Xiaoping Rui. Foundation and skill of ArcGIS Engine based on C \# language [M]. Beijing: Publishing House of Electronics Industry, 2015.

[2]Naixia Mou. The ArcGIS10 geographic information system tutorial from beginner to mastery [M]. Beijing: Surveying and Mapping Press, 2012.

[3]Fei Yang. Application of fire information system based on GIS technology [J]. China Science and Technology Information,2015.02.

[4]Zhixiang Xing. Urban fire rescue decision support system research review [J]. Journal of Safety Science and Technology.2011.11.

[5] Yanli Gao.Google Earth and the Sketch Up environment research methods to set Up the three-dimensional scene[J]. Science Mosaic.2012.05. 